\title{
NM_001013649.3 gene is down-regulated in human colorectal adenocarcinoma
}

\author{
QIN JIANG $^{1}$, CHUNLE ZHANG $^{2}$ and YAO CHEN ${ }^{1}$ \\ ${ }^{1}$ Department of Anatomy, Basic Medical and Forensic Medical Institute; \\ ${ }^{2}$ Department of Gastroenterology, West China Medical Center, Sichuan University, Chengdu, Sichuan 610041, P.R. China
}

Received April 27, 2011; Accepted August 16, 2011

DOI: $10.3892 / \mathrm{mmr} .2011 .563$

\begin{abstract}
The present study aimed to evaluate the expression of NM_001013649.3 in human colorectal adenocarcinoma tissue and its correlation with clinicopathological aspects of the disease. Through the combination of a cDNA subtractive library and cDNA microarray analysis, a differentially expressed sequence tag ES274081 was identified whose full-length cDNA sequence (NM_001013649.3) was obtained through BLASTn analysis on NCBI. Reverse transcription quantitative real-time PCR was used to assess the expression levels of NM_001013649.3 in cancer and adjacent normal colorectal tissues from 30 patients with colorectal adenocarcinoma. The mRNA levels of NM_001013649.3 were significantly lower in the cancer tissues compared to the adjacent normal colorectal tissues of 25 out of $30(83 \%)$ patients $(\mathrm{p}<0.005)$. Furthermore, the rate of down-regulation was significantly higher in moderately differentiated samples (21 out of $23 ; 91 \%$ ) compared to poorly differentiated samples $(3$ out of $6 ; 50 \%)(\mathrm{p}<0.05)$. The mRNA levels of $N M_{-} 001013649.3$ are significantly down-regulated in colorectal adenocarcinoma tissue, and this down-regulation is most likely correlated with the differentiation status of the cancer, indicating that NM_001013649.3 is potentially involved in the pathogenesis of colorectal adenocarcinoma.
\end{abstract}

\section{Introduction}

The prevalence of of colorectal adenocarcinoma has increased in recent years, and it is the second most common cancer and the second most common cause of cancer-related death worldwide (1-3). The causes and molecular mechanisms of colorectal adenocarcinoma are largely unknown, although they may involve multiple factors and multiple genes in

Correspondence to: Professor Yao Chen, Department of Anatomy, Basic Medical and Forensic Medical Institute, Sichuan University, Chengdu, Sichuan 610041, P.R. China

E-mail: xmxfh@263.net

Key words: human colorectal adenocarcinoma, tumor differentiation, mRNA, NM_001013649.3 multiple pathways. We have recently identified 86 differentially expressed sequences from human colorectal adenocarcinoma tissue compared with normal colorectal tissue, through cDNA subtractive library and cDNA microarray. In this study, we further investigate the mRNA expression levels in human colorectal adenocarcinoma tissues by using reverse transcription quantitative real-time (RT-qPCR) for one of the most significant differentially expressed sequences (ES274081) (4-6), whose full-length cDNA is NM_001013649.3 (NCBI), with the aim of assessing its association with clinicopathological aspects of human patients.

\section{Materials and methods}

Tissue samples. With informed consent from patients and approval from the Medical Research Ethics Committee at Sichuan University, fresh colorectal adenocarcinoma tissues and adjacent normal colorectal tissues ( $>5 \mathrm{~cm}$ away from the edge of the tumor, and no cancer cells under pathological examination) were collected from 30 patients with colorectal adenocarcinoma who were admitted to West China Hospital of Sichuan University. The pathology was confirmed for all samples. The tissues were immediately frozen and kept in liquid nitrogen until use.

RNA isolation. TRIzol reagent (Invitrogen, Carlsbad, CA, USA) was used for total RNA isolation according to the manufacturer's protocol. RNA yield was determined by measuring absorbency at $260 \mathrm{~nm}$ on a spectrophotometer, and total RNA was isolated according to the manufacturer's instructions. RNA integrity was judged by sharp 28S and 18S rRNA bands and 2:1 intensity ratio of the two bands (28S:18S) on 1\% agarose gel.

Synthesis of first-strand cDNA. The isolated RNA from each sample was treated with DNase I to remove genomic DNA contamination prior to reverse transcription. Total RNA $(1 \mu \mathrm{g})$ was reverse-transcribed using the M-MuLV reverse transcriptase kit (Fermentas, Canada) for first-strand cDNA synthesis according to the manufacturer's instructions. The synthesized cDNA was immediately used for quantitative real-time PCR, or kept at $-20^{\circ} \mathrm{C}$ until use.

Quantitative real-time PCR. Quantitative real-time PCR was performed for cDNA amplification using SYBR Premix 
Table I. Primers.

\begin{tabular}{ll}
\hline Gene & \multicolumn{1}{c}{ Primer sequences (5'-3') } \\
\hline NM_001013649.3 & F: ACGCAACCCAGACTATGAAGAG \\
& R: CACCTTCTCACTCACCTTTCCT \\
GAPDH & F: GGAAGGTGAAGGTCGGAGT \\
& R: TGAGGTCAATGAAGGGGTC \\
\hline
\end{tabular}

ExTaq (Takara, Japan) and primers listed in Table I based on the manufacturer's instructions and related international standards (7-9), using the Bio-Rad C1000 real-time system (Bio-Rad, USA). cDNA ( $2 \mu \mathrm{l})$ from $1 \mu \mathrm{g}$ RNA template was used for each PCR reaction. GAPDH was used as the internal control. The primers were designed in the open reading frame of NM_001013649.3 using Primer Premier 5.0 software.

Statistical analysis. All values are represented as means \pm SEM. Statistical analysis was performed using the Wilcoxon signed-rank test and Fisher's exact probabilities test with SPSS17.0. A P-value $<0.05$ was considered significant.

\section{Results}

The mRNA levels of NM_001013649.3 were found to be down-regulated in $83 \%$ of the tumor samples (25 out of 30 ), compared to those of the adjacent normal colorectal tissue samples. The expression levels of the samples are shown in Fig. 1, and the average expression levels in both the adenocarcinoma and normal colorectal tissues are shown in Table II. The mRNA expression levels of NM_001013649 in adenocarcinoma tissues were significantly lower compared to those of adjacent normal colorectal tissues (Wilcoxon signed rank test, $\mathrm{p}<0.005)$.

The effect of the patients' gender, age, Dukes' stage, and tumor differentiation on NM_001013649.3 expression is shown in Table III. The down-regulation rate was as high

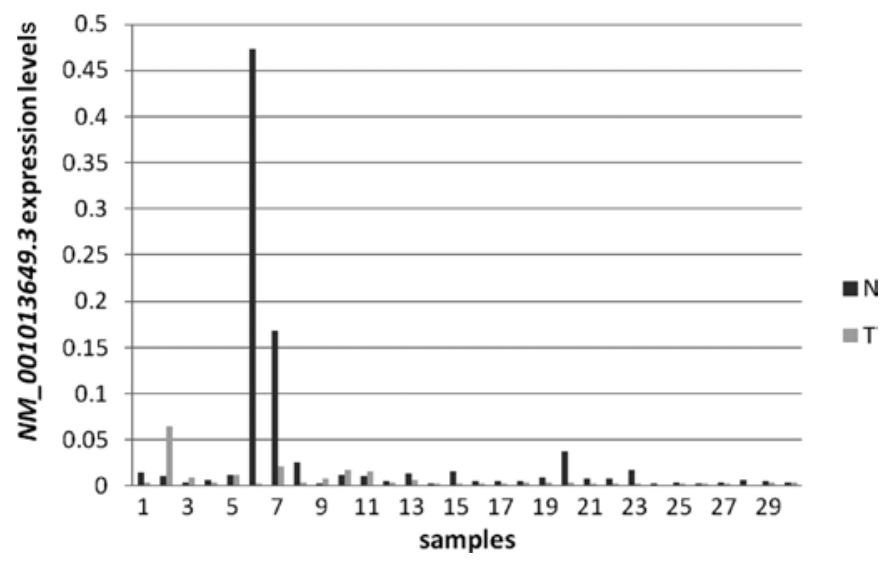

Figure 1. Expression levels of $N M_{-} 001013649.3$. T=374, $\mathrm{P}=0.004$ by Wilcoxon signed-rank test. T, colorectal adenocarcinoma sample; $\mathrm{N}$, matched normal sample.

as $91 \%$ in moderately differentiated samples (21 out of 23), but only $50 \%$ in poorly differentiated tissue samples (3 out of 6), indicating a significantly higher rate of down-regulation of NM_001013649.3 in moderately differentiated samples (Fisher's exact probability test, $\mathrm{p}<0.05$ ). One patient exhibited a markedly high expression level in the normal colorectal tissue (186.31-fold higher), compared to the adenocarcinoma tissue. Age, gender and tumor stage showed no significant effect on NM_001013649.3 mRNA expression.

\section{Discussion}

Through the cDNA subtractive library and cDNA microarray, one differentially expressed EST fragment was identified between tumor and normal colorectal tissues from 4 patients with colorectal adenocarcinoma in Dukes' A stage, with a reverse hybridization ratio of 2.472 (4). This EST fragment has a length of $735 \mathrm{bp}$, and its GenBank accession number is ES274081. Full-length cDNA was obtained by a BLASTn search on the NCBI website (4283 bp, GenBank accession

Table II. Expression levels of NM_001013649.3 in adenocarcinoma and normal tissues.

\begin{tabular}{lcccc} 
Name & Minimum & Maximum & Average & Standard error \\
\hline Normal & 0.00263 & 0.47323 & 0.3021930 & 0.8883944 \\
Adenocarcinoma & 0.00089 & 0.06425 & 0.0071123 & 0.1188477 \\
\hline
\end{tabular}

Table III. Effect of gender, age, Dukes' stage and tumor differentiation on NM_001013649.3 expression.

\begin{tabular}{|c|c|c|c|c|c|c|c|c|c|c|}
\hline & \multicolumn{2}{|c|}{ Gender } & \multicolumn{2}{|c|}{ Age (years) } & \multicolumn{3}{|c|}{ Dukes' stage } & \multicolumn{3}{|c|}{ Tumor differentiation } \\
\hline & Male & Female & $\geq 50$ & $<50$ & $\mathrm{~B}$ & $\mathrm{C}$ & $\mathrm{D}$ & Well & Moderately & Poorly \\
\hline \multicolumn{11}{|l|}{ Patients (no.) } \\
\hline Up-regulation & 1 & 4 & 3 & 2 & 1 & 3 & 1 & 0 & 2 & 3 \\
\hline Down-regulation & 16 & 9 & 23 & 2 & 5 & 14 & 6 & 1 & 21 & 3 \\
\hline
\end{tabular}


number NM_001013649.3). This gene is located on human chromosome 2p11.2 and encodes a human hypothetical protein LOC388969. To date, there have been no reports regarding the function of this gene $(10,11)$.

In the present study, we investigated the mRNA expression of the NM_001013649.3 gene using RT-qPCR in tumor and adjacent normal colorectal tissues from 30 patients with colorectal adenocarcinoma.

We found that the mRNA levels of NM_001013649.3 in the colorectal adenocarcinoma tissue were significantly lower than those in the adjacent normal colorectal tissue, and this down-regulation was observed in $83 \%$ of patients (25 out of 30 ), indicating potential involvement of this gene in the pathogenetic process of colorectal adenocarcinoma. Furthermore, the down-regulation was more frequently observed in moderately differentiated samples than in poorly differentiated samples $(\mathrm{p}<0.05)$, which may indicate a correlation of NM_001013649.3 gene expression with tumor differentiation. We did not find any effects of age, gender and tumor stage on NM_001013649.3 mRNA expression.

Further studies are required to investigate $N M \_001013649.3$ gene expression at the protein level and to probe the molecular pathways linking this gene and colorectal adenocarcinoma.

\section{Acknowledgements}

This study was supported by the Ministry of Education Fund for Returned Scholars from Overseas (2008101-3-3) and a grant from the Sichuan University for Stomatological Key Laboratories (SKLODSCU20090021).

\section{References}

1. Rougier P and Mitry E: Epidemiology, treatment and chemoprevention in colorectal cancer. Ann Oncol 14: 3-5, 2003.

2. Boyle P and Ferlay J: Cancer incidence and mortality in Europe, 2004. Ann Oncol 16: 481-488, 2005.

3. Zheng S and Cai SR: Colorectal cancer epidermiology and prevention study in China. Chin Ger J Clin Oncol 2: 72-76, 2003.

4. Chen Y, Zhang Y, Zhou Z, Wang G and Yi Z: Identification of differentially expressed genes in human colorectal adenocarcinoma. World J Gastroenterol 12: 1025-1032, 2006.

5. Chen Y, Zhang C, Shen YQ and Wang LC: Bioinformatics analysis and validation of the expressed sequences tag in human colorectal adenocarcinoma. Gastroenterol Res 2: 110-114, 2009.

6. Zhang $\mathrm{C}$ and Chen Y: Electronic cloning and validating of the suppression subtractive hybridization EST ES274070 of human colorectal adenocarcinoma. US Chin J Lymphol Oncol 6: 83-88, 2007.

7. Bustin SA, Benes V, Garson JA and Hellemans J: The MIQE guidelines: minimum information for publication of quantitative real-time PCR experiments. Clin Chem 55: 611-622, 2009.

8. Nolan T, Hands RE and Bustin SA: Quantification of mRNA using real-time RT-PCR. Nat Protoc 1: 1560-1581, 2006.

9. Pfaffl MW: A new mathematical model for relative quantification in real-time RT-PCR. Nucleic Acids Res 29: 2002-2007, 2001.

10. Brandenberger $\mathrm{R}$, Wei $\mathrm{H}$, Zhang S, et al: Transcriptome characterization elucidates signaling networks that control human ES cell growth and differentiation. Nat Biotechnol 22: 707-716, 2004.

11. Strausberg RL, Feingold EA, Grouse LH, et al: Generation and initial analysis of more than 15,000 full-length human and mouse cDNA sequences. Proc Natl Acad Sci USA 99: 16899-16903, 2002. 